\title{
Intact Retinal Pigment Epithelium Maintained by Nok Is Essential for Retinal Epithelial Polarity and Cellular Patterning in Zebrafish
}

\author{
Jian Zou, ${ }^{1}$ Kira L. Lathrop, ${ }^{1}$ Ming Sun, ${ }^{2}$ and Xiangyun Wei ${ }^{1,3}$ \\ ${ }^{1}$ Department of Ophthalmology, ${ }^{2}$ Center for Biologic Imaging, Department of Cell Biology and Physiology, and ${ }^{3}$ Department of Microbiology and Molecular \\ Genetics, University of Pittsburgh School of Medicine, Pittsburgh, Pennsylvania 15213
}

Within the vertebrate eye, the retinal pigment epithelium (RPE) juxtaposes with the retina, but how the RPE plays a role in retinal morphogenesis remains elusive. It has been shown that the loss of function of the polarity proteins, such as Nagie oko (Nok), disrupts RPE integrity and retinal lamination. However, it is unclear whether or not such defects are caused in a tissue-autonomous manner. Here, by taking advantage of the nok mutation, we have generated a transgenic model to restore the Nok function in the RPE, but not in the retina. With this model, we show that Nok is required for RPE integrity in a tissue-autonomous manner. However, proper retinal epithelial polarity does not require retinal expression of Nok before embryonic photoreceptor genesis; rather, it requires a Nok-mediated intact RPE. Interestingly, sporadic wild-type RPE donor cells are not sufficient to maintain proper retinal polarity. We further show that RPE-mediated retinal epithelial polarity underlies proper patterning of retinal ganglion cells and the cells of the inner nuclear layer. Nevertheless, during embryonic photoreceptor genesis, an intact RPE is not sufficient to maintain retinal epithelial polarity and retinal cellular pattern formation. Our results show that the subcellular architecture and cellular pattern formation of a tissue may be regulated by neighboring tissues through tissue-tissue interactions.

Key words: RPE; retina; cellular pattern formation; Nok; polarity; transgenesis

\section{Introduction}

In vertebrates, the optic cup originates from invagination of the optic vesicle. The outer layer of the cup becomes the retinal pigment epithelium (RPE), and the inner layer becomes the retina. During retinal neurogenesis, the retinal cells stratify into a layered structure. Each retinal layer is occupied by distinct types of cells that are positioned in specific geometric patterns (Dowling, 1970), but how such a retinal cytoarchitecture is formed during development is still not fully understood.

Because of the juxtaposition of the RPE with the retina, whether and how the RPE regulates retinal development has been an important research subject. Despite previous studies on this subject, the cellular and molecular mechanisms by which the RPE regulates retinal cellular pattern formation remain elusive. RPE ablation in transgenic mice showed that the RPE is needed to

Received Sept. 10, 2008; revised 0ct. 17, 2008; accepted 0ct. 31, 2008.

This work was supported by National Institutes of Health (NIH) Core Grant 5P30EY008098-17 and the following funds (X.W.): NIH Grant R01EY016099, University of Pittsburgh School of Medicine start-up fund, and Research to Prevent Blindness Career Development Award. We are grateful to Dr. Pamela Raymond and Lynne Sunderman for critical editing of this manuscript, to Dr. Friedrich Beermann for providing Fugu tyrosinase gene promoter, to Drs. Jan Wijnholds and Penny Rashbass for anti-Crumbs antibodies, and to Dr. Paul Linser for providing the anti-carbonic anhydrase antibodies. We also thank Drs. Donna Stolz and Simon Watkins for providing the transmission electron microscopy facilities at the Center for Biologic Imaging at University of Pittsburgh School of Medicine. Dr. Richard Bilonick provided assistance in statistical analysis.

Correspondence should be addressed to Xiangyun Wei, University of Pittsburgh, 3501 Fifth Avenue, BST3, Room 5060, Pittsburgh, PA 15213. E-mail: weix@upmc.edu.

DOI:10.1523/JNEUROSCI.4333-08.2008

Copyright $\odot 2008$ Society for Neuroscience $\quad 0270-6474 / 08 / 2813684-12 \$ 15.00 / 0$ maintain the survival of the retina (Raymond and Jackson, 1995). In vitro culture of dissociated chicken retinal cells suggested that the RPE may secrete unknown factor(s) to regulate retinal lamination (Vollmer et al., 1984; Rothermel et al., 1997; Nakagawa et al., 2003). In addition, blastomere transplantation experiments revealed that the mosaic eyes (moe) and nagie oko (nok) genes function in a non-cell-autonomous manner in patterning retinal cells (Jensen et al., 2001; Wei and Malicki, 2002; Zolessi et al., 2006). Although these experiments indicated that retinal development requires extrinsic regulations, each of these studies has particular limitations on revealing the mechanisms involved: the absence of the RPE in the transgenic mouse models made it difficult to analyze direct physical interactions between the RPE and the retina, and it was hard to distinguish the trophic function of RPE from its other functions; the in vitro systems may not fully represent the in vivo conditions; and blastomere transplantations in zebrafish generated uncontrollable distribution of donor cells in the host RPE and retina, making it difficult to determine unequivocally whether the RPE-retina or the retina-retina interactions are essential for the proper patterning of retinal cells. Thus, a different in vivo experimental approach is needed to provide additional insights into the RPE-retina interactions.

To achieve this goal, we took advantage of the zebrafish nok mutation and generated a transgenic zebrafish model (pt106) to restore the Nok functions in the RPE but not in the retina. The nok gene encodes a member of the membrane-associated guanylate kinase protein family (Wei and Malicki, 2002; Funke et al., 
2005). Loss of Nok function causes patchy RPE and retinal lamination defects (Wei and Malicki, 2002). Because the loss of Nok function affects the development of both the RPE and the retina, the tissue-specific restoration of the RPE by transgenic Nok expression in pt106 will provide a unique in vivo system to answer certain questions about RPE-retina interactions: Does the loss of nok function cause the retinal and RPE defects in a tissue-autonomous manner? How does an intact RPE regulate retinal development at the cellular and subcellular levels? We demonstrate for the first time that the maintenance of retinal epithelial polarity requires an intact wild-type RPE but not a few sporadic wild-type donor RPE cells. This RPEmediated retinal epithelial polarity is essential for cellular pattern formation during retinal neurogenesis.

\section{Materials and Methods}

Generation of the pt106 and pt104 embryos. We used the Fugu tyrosinase promoter to drive a transgenic expression of a wild-type Nok gene in the RPE. The Fugu tyrosinase promoter was amplified from $\mathrm{pF} 3 \mathrm{xho5}^{\prime}$ (a gift from Dr. Friedrich Beermann, Swiss Institute for Experimental Cancer Research, Epalinges, Switzerland) by PCR and used to replace the EF1 $\alpha$ promoter between the ApaI and BamHI sites of the Tol2 transgenesis construct pT2KXIG $\Delta$ in (Camacho-Hübner et al., 2000; Zou et al., 2006). The wild-type Nok ORF, including a TGA stop codon, was inserted between the Fugu tyrosinase promoter and green fluorescent protein (GFP) ORF by an AgeI and FseI restriction ligation. The resulting construct (pTol2-ftyp-Nok-GFP) (see Fig. 2A) was used for transgenesis according to the established Tol2 methodology (Zou et al., 2006). To make transgenic founder fish, $\sim 10-20 \mathrm{pg}$ of pTol2-ftyp-Nok-GFP along with 25-50 pg of Tol2 transposase mRNA were coinjected at one- to four-cell stages into embryos obtained from crosses between $n o k^{m 520}$ carriers. Seven transgenic positive founder fish were outcrossed with $n o k^{m 520}$ heterozygous fish. Transgenic positive $\mathrm{F}_{1}$ fish were identified by a PCR genotyping analysis using primers $5^{\prime}$-aacaaaaatgatgactttg- $3^{\prime}$ and $5^{\prime}$-tcagcgcagccaggatgaag- $3^{\prime}$. To screen for $F_{1}$ individuals that carry a genome-integrated transgene with a good RPE rescuing capability, individual $\mathrm{F}_{1}$ transgenic fish that are also heterozygous for $n o k^{m 520}$ were crossed with regular $n o k^{m 520}$ heterozygous fish for examination of their $\mathrm{F}_{2}$ progeny. Of 74 transgenic and $n o k^{m 520} \mathrm{~F}_{1}$ carriers, we identified two $\mathrm{F}_{1} \mathrm{~s}$ that rescued the patchy RPE defect in $50 \%$ of the mutant embryos, indicating that these two fish carried a single copy of the transgene. One line was further characterized in this study and is designated pt $106^{\text {bing-33. }}$. The transgenic mutant embryos produced from the crosses between pt106 $6^{\text {bing-33 }}$ and regular nok $k^{m 520}$ carries were named pt 106 embryos.

To generate a stable transgenic line that express GFP in an ubiquitous manner, we injected Tol 2 transposase mRNA along with pT2KXIG $\Delta$ in, which contains a GFP open reading frame downstream of the $\mathrm{EF} 1 \alpha$ promoter, into $\mathrm{AB}$ wild-type embryos. This line was named pt104. Care of experimental animals was in accordance with University Pittsburgh guidelines.

In situ hybridization. In situ hybridization analyses of transgenic Nok expression and zebrafish tyrosinase-related protein 1 (TYRP1) were performed according to a previous publication (Zou et al., 2006).

The generation of rabbit anti-Nok $C$ terminal polyclonal antibody. The C-terminal region of $\mathrm{Nok}^{505-703}$ (from amino acid 505 to amino acid 703) was PCR amplified and cloned into the His tag expression vector pET32a + (Novagen) between EcoRI and HindIII. The construct was transformed into BL21 competent cells (Invitrogen) and used to express the Nok ${ }^{505-703}$-His fusion protein. Two milligrams of $\mathrm{Nok}^{505-703}$-His was purified with a His-trap column (GE Healthcare) and used to immunize rabbits using the service provided by Proteintech Group. Antibodies that only recognize Nok downstream of the $m 520$ mutation site were affinity purified using a Aminolink Plus immobilization affinity column (Pierce) that was conjugated with $1 \mathrm{mg}$ of GST-Nok ${ }^{547-703}$ fusion protein [expressed in the pGex-5x-1 system (GE Healthcare)].

Immunohistochemistry. Immunohistological analyses were performed using the procedure and reagents as described previously (Wei et al., 2006b), except that the embryos were fixed at room temperature (RT) for 30 min for anti-Nok ${ }^{547-703}$ immunostaining.
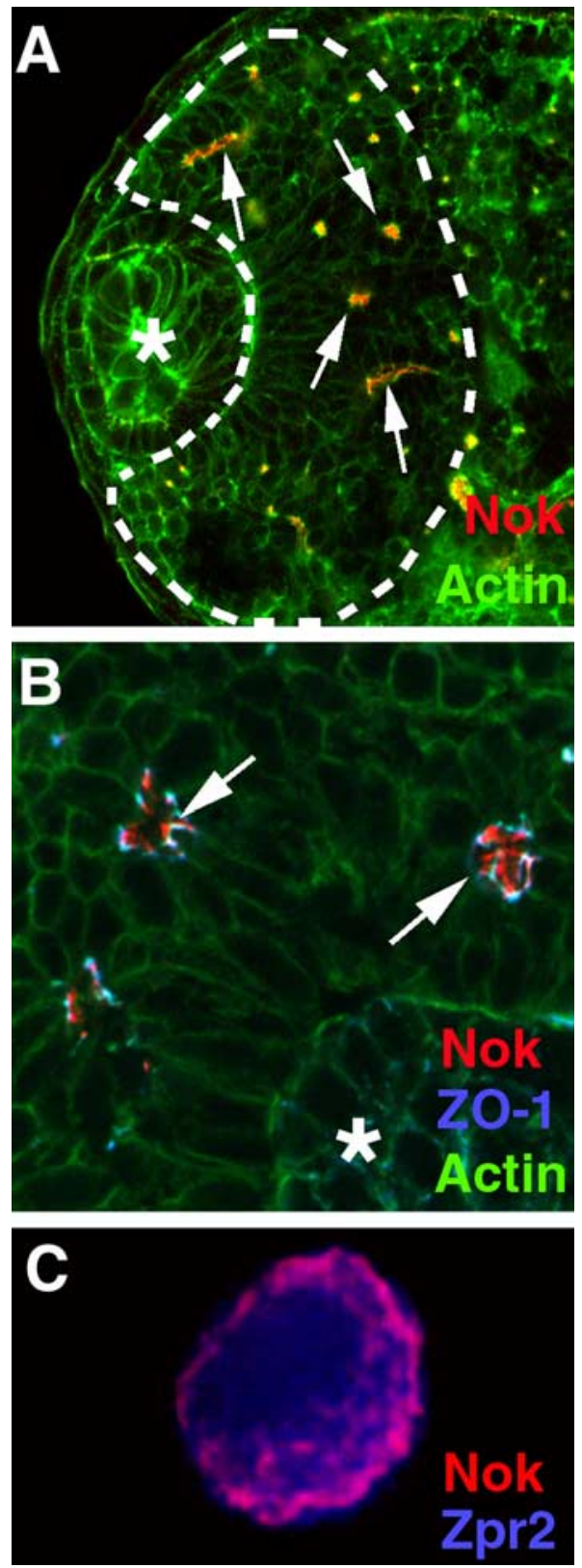

Figure 1. The nok gene is expressed in both the neural retina and the RPE. $A, B$, At $35 \mathrm{hpf}$, Nok (red; arrows) localizes to the vicinity of ectopic adherens junction clusters marked by Z0-1 (blue) and actin bundles (green) in the interior of the $\mathrm{N}$-cad ${ }^{m 117}$ mutant retinal neuroepithelium. The lens is indicated by an asterisk. The dashed line in $\boldsymbol{A}$ demarks the boundary of the retina. $\boldsymbol{B}$ is shown at a higher magnification than that in $\boldsymbol{A}$. $\boldsymbol{C}$, A cell, dissociated from $72 \mathrm{hpf}$ eyes, displays signals for both Nok (red) and RPE-specific marker zpr2 (blue).

Blastomere transplantation. Wild-type pt104 donor blastomeres were transplanted into either regular nok ${ }^{m 520}$ mutant or pt 106 host embryos at 3-4 hours postfertilization (hpf) using the standard transplantation technique (Ho and Kane, 1990). The resulting mosaic embryos were raised in egg water at $28.5^{\circ} \mathrm{C}$ until $36 \mathrm{hpf}$ before fixation with $4 \%$ paraformaldehyde at room temperature for $2 \mathrm{~h}$. Fixed embryos were subjected to standard immunohistochemical analyses for examination of retinal polarity phenotypes.

Immunohistochemical analysis of dissociated RPE cells. Wild-type embryos were raised in E3 egg water with $0.003 \%$ of pigmentation-blocking chemical 1-phenyl-2-thiourea (PTU) (Sigma-Aldrich) until $72 \mathrm{hpf}$. Forty eyes were removed from these embryos and digested with $200 \mu \mathrm{l}$ of TrypLE Express (Invitrogen; containing amphotericin B and penicillinstreptomycin) at RT for $30 \mathrm{~min}$. The digestion was stopped by adding 20 

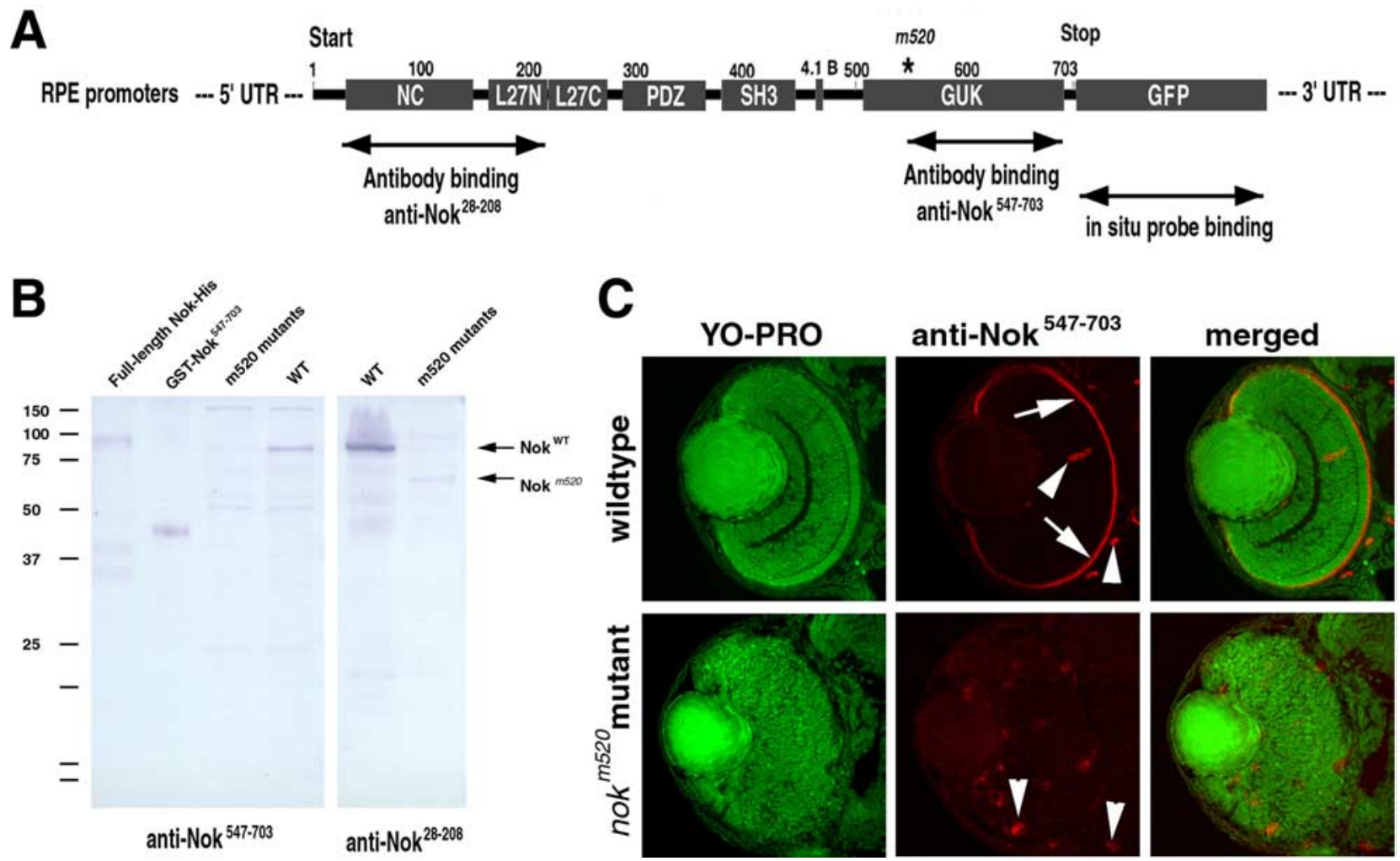

Figure 2. Strategies for transgenic expression of Nok in the RPE and for selection of desired transgenic zebrafish lines. $\boldsymbol{A}$, The promoter of Fugu tyrosinase gene was used to direct pigment cell-specific expression of the full-length wild-type nok gene. Individual protein domains are indicated with gray boxes. A GFP coding region was inserted between the stop codon of the nok gene and the SV40 poly(A) signal-containing 3'-untranslated region. This GFP sequence serves as the target of a GFP antisense probe for in situ analyses. The Nok C-terminal region downstream of the m520 nonsense mutation site was used to make an affinity column to purify the anit-Nok ${ }^{547-703}$ polyclonal antibodies. Anti-Nok ${ }^{28-208}$ antibodies (generated in a previous study) (Wei and Malicki, 2002) recognize both the full-length Nok and the truncated $\mathrm{Nok}^{\mathrm{m} 520}$. B. Western blot analyses showed that anti-Nok ${ }^{547-703}$ antibodies recognized the full-length Nok protein from wild-type fish samples (WT) and the recombinant Nok-His and GST-Nok ${ }^{547-703}$ fusion proteins expressed in Escherichia coli. Anti-Nok ${ }^{547-703}$ antibodies do not recognize the truncated Nok ${ }^{\mathrm{m} 520}$ mutant protein ( $m 520$ mutants). A few weaker bands indicate the existence of nonspecific reactions of the antibodies to some fish proteins. The positions of full-length and $m 520$ mutant Nok bands were indicated with arrows on the right. C, An immunohistochemical analysis demonstrated that the anit-Nok ${ }^{547-703}$ antibodies recognize full-length Nok in wild type at $72 \mathrm{hpf}$. Nok localizes to the apical regions of the RPE and the retina (arrows). The arrowheads indicate the nonspecific staining of cells of an unknown class, which appear to be randomly distributed in the brain and retina. In mutant retinas, only nonspecific staining was observed (arrowheads). Nuclear dye YO-PRO was used to visualize the overall shape of the eyes.

$\mu \mathrm{l}$ of (10\%) FBS and chilled to $4^{\circ} \mathrm{C}$. The supernatant of the digest was further microcentrifuged at $2000 \mathrm{rpm}$ for $5 \mathrm{~min}$ to collect the dissociated cells. The cell pellet was resuspended with $100 \mu \mathrm{l}$ of $1 \times \mathrm{PBS}$ at $4^{\circ} \mathrm{C}$ and spread on a poly-L-lysine-coated slide (Thermo Fisher Scientific), followed by an incubation of $1 \mathrm{~h}$ at $4^{\circ} \mathrm{C}$. The cells adhered to the slide were then fixed with $2 \%$ paraformaldehyde for $1 \mathrm{~h}$. To determine whether or not RPE cells express Nok, the slide was immunostained with the rabbit polyclonal anti-Nok ${ }^{28-208}$ antibodies (1:200) (Wei and Malicki, 2002) and the mouse monoclonal zpr2 antibody (1:200; ZFIN), which recognizes an RPE-specific antigen. Expression of Nok in zpr2-positive cells were examined under confocal microscopy.

\section{Results}

The nok gene is expressed in both the RPE and the retina

A display of a heritable developmental defect in a tissue does not necessarily mean that the tissue expresses a particular mutant gene. To restore the RPE integrity in $n o k^{m 520}$ by expressing a wild-type nok transgene, we first need to confirm that the nok gene is indeed expressed in the RPE. Previously, we revealed that the Nok protein localizes to the interface between the retinal neuroepithelium and the RPE (Wei and Malicki, 2002). However, because the apical surfaces of the two tissues are in close contact at early epithelial stages, the limited resolution of conventional light microscopy does not allow definitive assertion about the tissue expression pattern of Nok. In fact, another study has since suggested that Nok was expressed in the RPE but not in the retina (Jensen and Westerfield, 2004). To unambiguously confirm that both the RPE and the retina express Nok, we need to spatially separate the apical surfaces of the two tissues when examining the expression patterns of Nok. We thus first analyzed the distribution of the Nok proteins in the $35 \mathrm{hpf} \mathrm{N}$-cad ${ }^{m 117}$ mutant retinas, at which the apical surface of the retinal neuroepithelial cells localizes ectopically to the interior of the retina, three to seven cells away from the RPE (Erdmann et al., 2003). Indeed, we found that Nok localizes to the interior of the $N-$ cad $^{m 117}$ retina, demonstrating that the retina expresses Nok (Fig. 1A,B). To determine whether Nok is also expressed in the RPE, we chose to analyze dissociated individual RPE cells but not the RPE tissue in $\mathrm{N}$-cad ${ }^{m 117}$ to avoid the potential signal interference from the adjacent choroidal cells. Indeed, Nok is expressed in the RPE (Fig. 1C). The expression of Nok in both the RPE and the retina makes it legitimate to examine the effects of RPE restoration on retinal development in $n o k^{m 520}$ by expressing a wildtype transgenic nok gene in the RPE.

Transgenic expression of Nok in the RPE rescues the patchy RPE defect caused by the $\boldsymbol{n o k}^{m 520}$ mutation in a tissueautonomous manner

To achieve an RPE-specific transgenic expression of Nok in the eye, we used the Fugu tyrosinase gene promoter to express a wild-type transgenic nok gene in the nok ${ }^{m 520}$ mutant background 

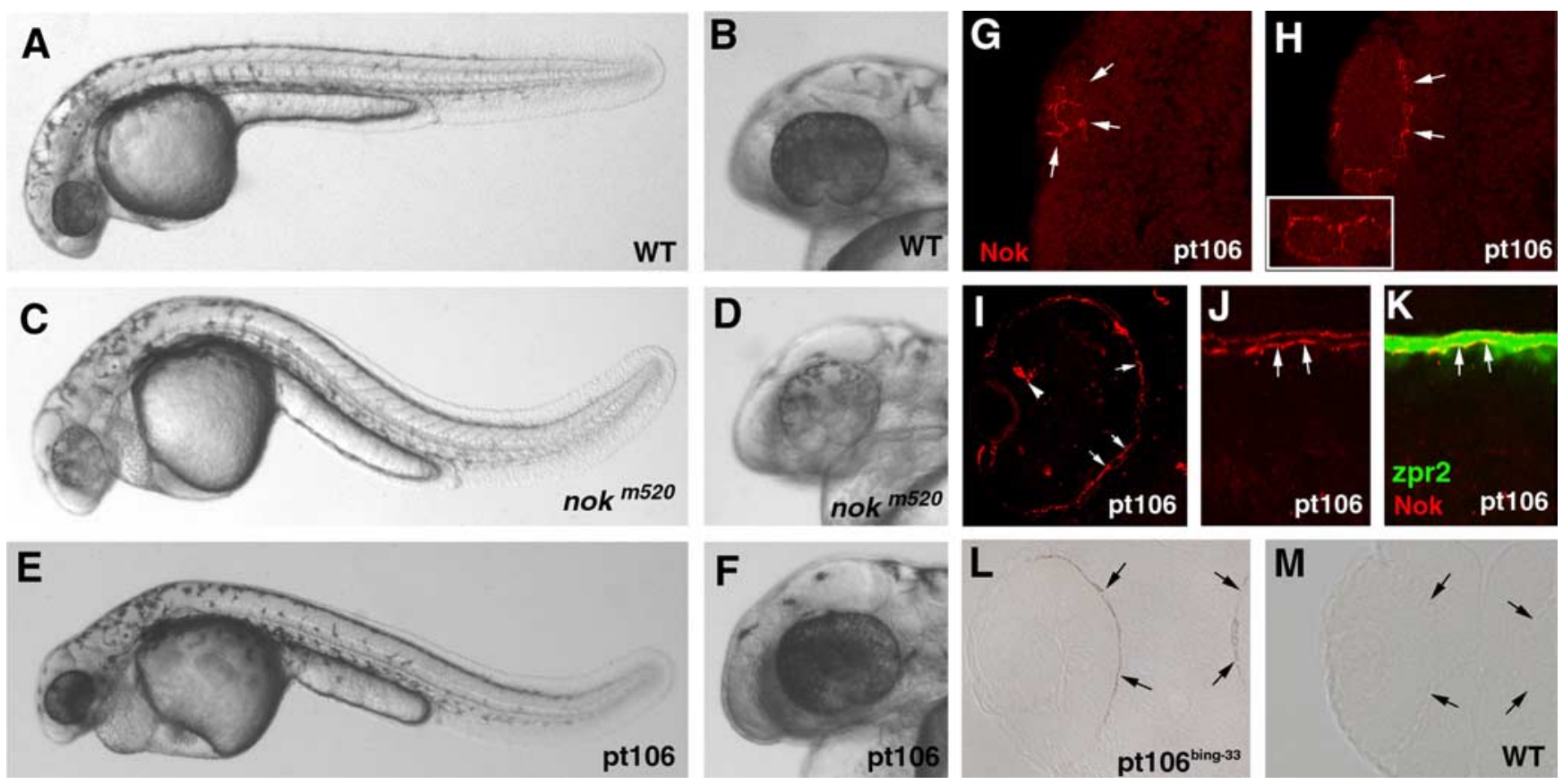

Figure 3. Transgenic expression of Nok in the RPE of pt 106 is sufficient to rescue the patchy RPE defect caused by the nok ${ }^{m 520}$ mutation. $\boldsymbol{A}-\boldsymbol{F}$, The expression of transgenic Nok in pt106 $(\boldsymbol{E}, \boldsymbol{F})$ restores the RPE integrity to a level indistinguishable from that of wild-type (WT) RPE $(\boldsymbol{A}, \boldsymbol{B})$. The phenotypes of a curled body axis and a paracardiac edema are still present in pt $106(\boldsymbol{E})$ as in nok ${ }^{m 520}$ mutants $(\boldsymbol{C})$ at $36 \mathrm{hpf}$. $\boldsymbol{B}, \boldsymbol{D}$, and $\boldsymbol{F}$ show magnified eye regions in $\boldsymbol{A}, \boldsymbol{C}$, and $\boldsymbol{E}$, respectively. $\boldsymbol{G}, \boldsymbol{H}$, Anti-Nok ${ }^{547-703}$ antibody visualized peripheral localization of transgenic $\mathrm{Nok}$ (red) in the eyes of pt 106 at $33 \mathrm{hpf}$ (arrows). $\boldsymbol{G}$ and $\boldsymbol{H}$ show images of the same embryo at different confocal optical sections. The inset shows a magnified RPE area that expresses transgenic Nok (red). $\boldsymbol{I}-\boldsymbol{K}$, Transgenic Nok (red; arrows) localizes to the RPE that was visualized with RPE-specific marker zpr2 (green) at $72 \mathrm{hpf}$. The arrowhead indicates the nonspecific staining of the anti-Nok ${ }^{547-703}$ antibody (Fig. 2). I shows the overview of an entire eye. $L, M$, Using a GFP riboprobe that recognizes the mRNA of the transgenic nok gene but not the endogenous nok gene (Fig. 2), an in situ hybridization analysis of 33 hpf PTU-treated wild-type pt $106^{\text {bing-33 }}$ embryos confirmed that the transgene is expressed in the RPE (arrows) but not in the neural retina $(\boldsymbol{L})$. A control regular wild-type embryo showed no detectable signal $(\boldsymbol{M})$.

(Fig. 2A) (Zou et al., 2006). Of 74 distinct $\mathrm{F}_{1}$ transgenic fish lines, we established one stable transgenic model (pt106) that is homozygous for the $n o k^{m 520}$ mutant gene and heterozygous for the wild-type nok transgene. In pt106, the expression of the transgenic nok gene restores RPE integrity, but the developmental defects of a curled body axis and paracardiac edema remain the same as that of regular $n o k^{m 520}$ mutant embryos (Fig. $3 A-F$ ). To verify the specificity of the transgenic expression, we generated polyclonal antibodies that recognize the full-length Nok protein but not the truncated endogenous $m 520$ mutant Nok protein (Fig. 2). Using the antibodies, we determined that the transgenic Nok protein is expressed in the RPE (Fig. 3G-K). An in situ hybridization analysis further confirmed that the transgene is expressed in the RPE but not in the retina (Figs. $2 A, 3 L, M$ ). Thus, Nok maintains RPE integrity in a tissue-autonomous manner.

\section{Retinal lamination is mostly restored in pt106}

To analyze the effect of the restoration of RPE integrity on retinal cellular pattern formation in pt106, we performed histological analyses of retinal structure at 4.5 or $5 \mathrm{~d}$ postfertilization (dpf). We found that retinal lamination is dramatically recovered in pt106 (Fig. 4A). Specifically, we found that retinal ganglion cells, Muller cells, and Lin7-positive cells (mainly bipolar cells, amacrine cells, and some ganglion cells that are adjacent to the inner plexiform layer) (Wei et al., 2006a) localize to proper cellular layers in pt106 (Fig. 4B,C). The processes of bipolar cells and Muller cells are also oriented radially in pt106 as in wild type, except that apical processes from Muller cells did not appear to extend to the outer nuclear layer (Fig. $4 B, C$ ). The integrity of the inner and outer plexiform layers, as revealed by actin staining, is also greatly improved (Fig. $4 B-D$ ). However, green/red double cones were found in all three layers, with only $\sim 50 \%$ of them in the photoreceptor layer. In pt106, these double cones are not as elongated as their counterparts in wild type, indicating their morphological defects at the cellular level. In addition, rods and blue and UV cones are also aberrantly positioned, similar to double cones in pt106 (data not shown). Interestingly, we rarely saw photoreceptor cells in the outer one-half of the inner nuclear layer in which the cell bodies of bipolar cells localize (Fig. 4D). We also found no apparent outer limiting membrane (OLM)like structure in pt106 at $4.5 \mathrm{dpf}$ (Fig. $4 B-D)$. Thus, the rescue of RPE defects by RPE expression of Nok in pt106 has a dramatic positive effect on retinal cytoarchitecture, leading to the proper positioning of several major retinal cell types (except for photoreceptors) and the overall structural recovery of inner and outer plexiform layers.

\section{Positive correlation between apical cell division and the patterning of retinal cells}

Although complex, the process of cellular patterning for a given postmitotic cell can be divided into two general steps. First, the cell needs to migrate to a proper place if its birth place is not its final destination. Second, stabilizing mechanisms are used to ensure that the cell makes proper contacts with its neighbor cells and does not move away from its destination. Here, the differential effects of pt106 transgenic Nok expression on the positioning of ganglion cells, inner nuclear layer cells, and photoreceptors may provide an opportunity to analyze how the cellular patterning process goes wrong in $n o k^{m 520}$ retinas. The results will provide insights into the mechanisms by which retinal cells are normally patterned in wild type.

To dissect the cellular basis of the retinal pattern formation, 
we first analyzed the distribution of M-phase nuclei at distinct developmental stages when ganglion cells, inner nuclear layer cells, or photoreceptors are each predominantly generated $(\mathrm{Hu}$ and Easter, 1999). The rationale for this analysis is that, during neurogenesis, some cell divisions are final cell divisions, after which those cells become postmitotic; therefore, the locations of M-phase nuclei will provide information about the start sites of postmitotic cell migrations (although we recognize that this analysis does not distinguish final cell divisions from other cell divisions). To more accurately define the start sites of postdivision cell migrations, we calculated the percentage of apical cell division events by counting the nuclei in late M-phase, namely, in metaphase, anaphase, or telophase (some cells in prophase are still moving toward the apical surface, and therefore, their locations do not accurately represent the start sites of postdivision migration, so these were not included). We found that, as in wild type, the majority of retinal cell divisions occur apically in pt106 when ganglion cells and inner nuclear cells are being generated (Fig. $5 A, B, D$ ). During embryonic photoreceptor genesis, the percentage of apical cell divisions declines in pt106 (although still higher than that in regular nok $k^{m 52}$ mutant retinas) (Fig. 5C,D). Because retinal ganglion cells and inner nuclear cells are patterned more properly than photoreceptors in pt106 (Fig. 4), the above observations reveal a positive correlation between the frequency of apical localization of cell divisions and the degree of proper cellular patterning. This correlation suggests that apical localization of final cell divisions may play an essential role in proper postmitotic cellular patterning.

In addition to the starting sites of cell migration, the migratory directions of postmitotic cells also influence the final destinations of cells. To analyze the features of cell movements during retinal neurogenesis in wild type and in $n o k^{m 520}$, we examined nuclear movements in living embryos. Consistent with previous findings (Hinds and Hinds, 1974; Das et al. 2003; Baye and Link, 2007), we found that the majority of retinal interphase nuclei move radially along the apicobasal axis in wild type (Fig. 6A,C; supplemental movie 1 , available at www.jneurosci.org as supplemental material). However, in mutant retinas, many nuclei did not move radially; many round nuclei moved perpendicularly to the apicobasal axis of the retinal neuroepithelium (Fig. $6 B, D$ ). In addition, many cells in the mutant retinas move toward the interior of the retina before cell division: Cells in the inner one-half of the retina move apically to the interior to divide, whereas cells in the outer one-half of the retina move basally to the interior to divide (supplemental movie 2, available at www.jneurosci.org as supplemental material). Thus, the retinal epithelium is split in two in $n o k^{m 520}$,
WT
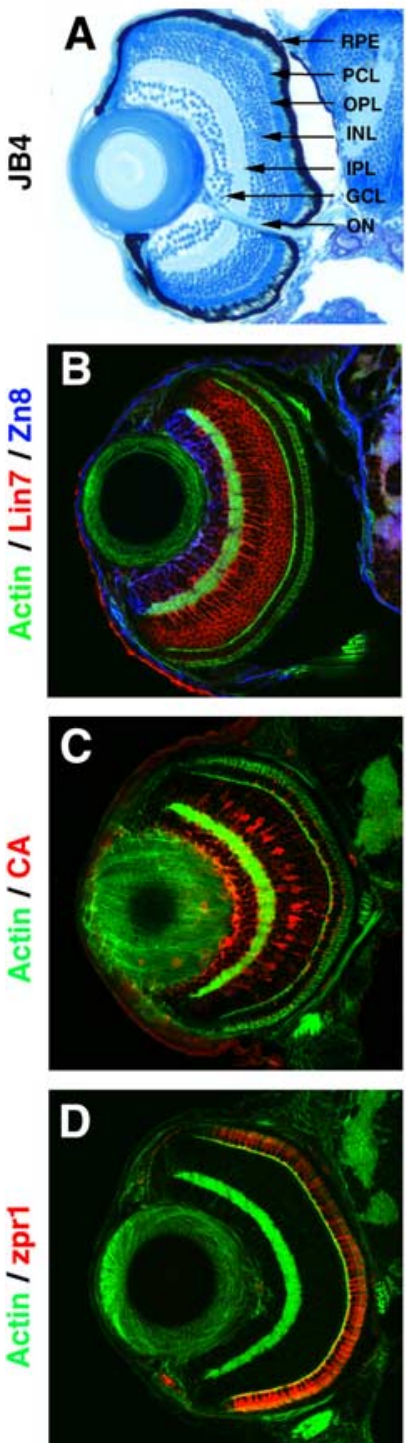

Figure 4. The retinal lamination is mostly restored in pt106. A, A JB4 histological analysis revealed a laminar cellular structure in the $5 \mathrm{dpf}$ pt106 retinas. PCL, Photoreceptor cell layer; INL, inner nuclear layer; OPL, outer plexiform layer; IPL, inner plexiform layer; $\mathrm{GCL}$, ganglion cell layer; $0 \mathrm{~N}$, optic nerve. $\boldsymbol{B}$, Unlike in nok ${ }^{m 520}$ mutants, ganglion cells (zn8staining in blue) and Lin7-positive cells (red) are positioned properly in pt106 as in wild type at $4.5 \mathrm{dpf}$. C, Muller cells (red; CA staining for carbonic anhydrase) are cellular layers in pt106 at $4.5 \mathrm{dpf}$. Phalloidin staining for actin (green) highlights the nearly wild-type-like organization of the inner and outer interplexiform layers in pt106 (B-D).

with each one-half displaying reversed directions of cellular movements.

Together, we infer that the ectopic interior localization of cell divisions and aberrant directions of cell migrations may directly contribute to the eventual cellular patterning defect in $n o k^{m 520}$ mutant retinas.

Proper early retinal epithelial polarity requires Nok expression in the RPE but not necessarily in the retina Because the localization of M-phase nuclei reflects how the epithelium polarizes (Hinds and Hinds, 1974), the above results suggest that the rescue of RPE defects by RPE expression of Nok restores the polarity of the retinal epithelium in pt106 before embryonic photoreceptor genesis. To confirm this, we next examined additional epithelial polarity markers ZO-1 and adherens junction-associated actin bundles. Indeed, unlike in $n o k^{m 520}$ (Fig. 

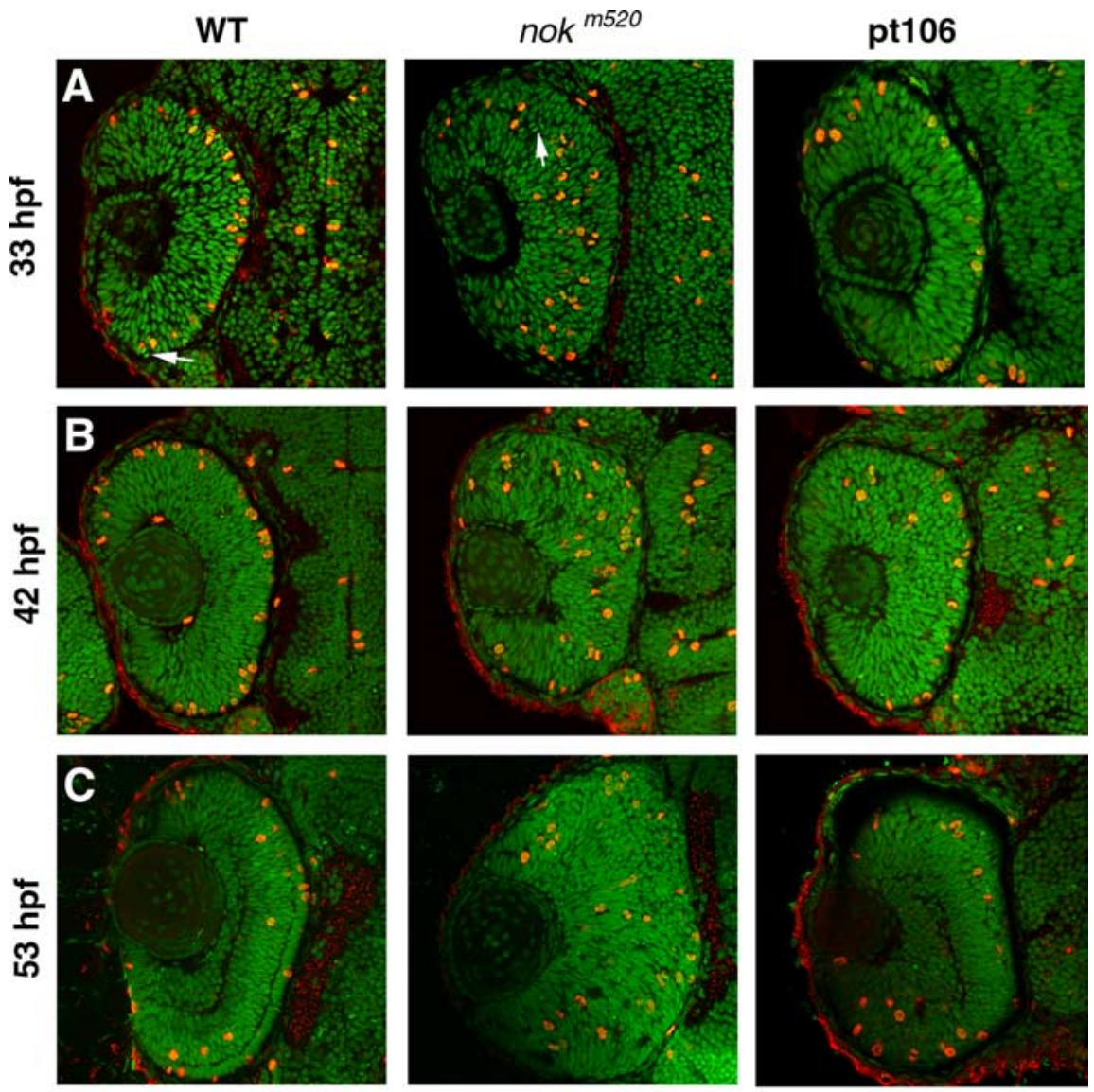

D

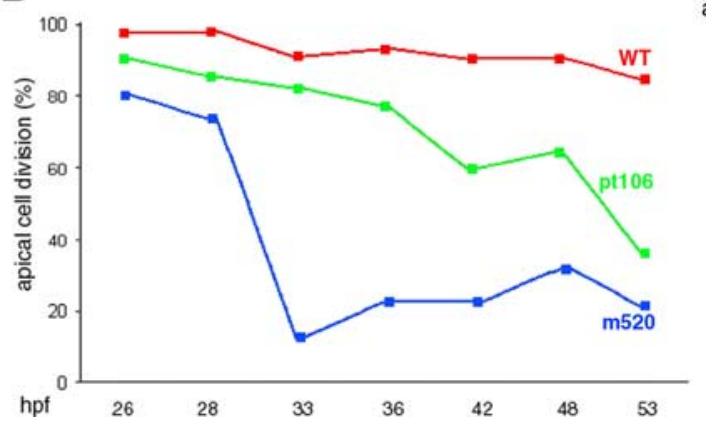

Total nuclei counted in metaphase, anaphase, or telophase

\begin{tabular}{llll} 
hpf & WT & pt106 & m520 \\
\hline 26 & 217 & 187 & 222 \\
28 & 170 & 132 & 197 \\
33 & 214 & 274 & 387 \\
36 & 332 & 259 & 382 \\
42 & 228 & 264 & 441 \\
48 & 143 & 144 & 182 \\
53 & 236 & 190 & 230
\end{tabular}

Figure 5. At early stages of embryonic retinal neurogenesis, cell divisions occur predominantly at the apical region of the retina in pt106 as in wild type. $\boldsymbol{A}-\boldsymbol{C}$, The localization of M-phase nuclei in wild type, nok ${ }^{\mathrm{m} 520}$, and pt106 at 33, 42, and 53 hpf were revealed by phospho-histone 3 staining (red) or YO-PRO nuclear staining (green, for chromatin condensation). The arrows indicate that some nuclei in late cell division stages (anaphase and telophase) display weak or no PH3 staining. D, A chart to show the percentage of cell division at the apical region of the retina at different developmental stages in pt106, wild type, and nok ${ }^{m 520}$. The numbers of nuclei in metaphase, anaphase, or telophase at each stage are presented in the table on the right, as determined by $\mathrm{PH} 3$ staining or by the morphological condensation of chromatin as revealed by $\mathrm{YO}-\mathrm{PRO}$ staining.

$7 B)$, these two markers are primarily positioned at the apical surface of the retina in pt106 and wild-type retinal epithelia (Fig. $7 A, C)$. Thus, transgenic expression of Nok in the RPE can sufficiently restore the proper polarity of retinal neuroepithelium at early stages of retinal development in pt106.

We next investigated whether or not retinal expression of Nok plays an active role in the polarization of the retinal epithelium. We transplanted wild-type retinal donor cells to either regular $n o k^{m 520}$ or pt 106 hosts. To visualize donor cells, we first generated a stable transgenic fish line (pt104) that expresses GFP under the ubiquitous EF1 $\alpha$ promoter in a wild-type genetic background. The transgenic GFP expression allows a visualization of live do- nor cells, avoiding the signal interference from dead donor cells when labeled with conventional nondegradable dextran conjugates (Catalano et al., 2007). We found the apical markers Nok and ZO-1 of the wild-type pt104 donor cells localized apically in pt106 host retinas but internally in $n o k^{m 520}$ host retinas (Fig. $8 A, B$ ). Because the only genetic difference between the regular nok ${ }^{m 520}$ and pt106 host eyes is that pt106 RPE expresses the wild-type transgenic nok gene, the above results suggest that the retinal expression of Nok is not sufficient to maintain proper polarity of the donor retinal epithelial cells at early developmental stages; instead, Nok expression in the RPE, which restores the RPE integrity, is required and sufficient to maintain proper retinal epithelial polarity.

An intact RPE but not sporadic wildtype RPE cells is required to restore the mutant retinal epithelial polarity defect in $\boldsymbol{n o k}^{\mathrm{m} 520}$

Restoration of RPE integrity and retinal epithelial polarity in pt106 raises interesting questions: Is RPE integrity important for maintaining retinal epithelial polarity? Or can sporadic wild-type RPE cells be sufficient to maintain retinal epithelial polarity? To analyze the correlation of the RPE integrity and retinal polarity defects in $n o k^{m 520}$, we first examined the temporal course of the disintegration of $n o k^{m 520}$ RPE with a zebrafish TYRP1 riboprobe as an early RPE integrity indicator (Zou et al., 2006). We found that, at $24 \mathrm{hpf}$, the RPE in $n o k^{m 520}$ is as intact as in wild type (Fig. 9A). At $26 \mathrm{hpf}$ in $n o k^{m 520}$, the retinal epithelial polarity is as normal as in wild type (Fig. 9B). At $\sim 28$ hpf when the polarity of retinal epithelium starts to deteriorate (data not shown), the RPE in $n o k^{m 520}$ begins to lose its intactness, as indicated by the presence of occasional gaps in the epithelial sheet (Fig. 9A). The disruption of RPE integrity becomes more severe and apparent $\sim 32 \mathrm{hpf}$, when retinal polarity is severely perturbed (Fig. 9A). Thus, the loss of retinal epithelial polarity is linked to the disruption of the RPE integrity in a tight temporal manner.

In the nok mutant retinas at $48 \mathrm{hpf}$, retinal ganglion cells prefer to accumulate at regions adjacent to the basement membrane of the retina or to apical retinal regions that lack RPE cells (Zolessi et al., 2006) (Fig. 9C). Zolessi et al. (2006) proposed that the RPE might play a role in blocking the attracting influence from the Bruch's membrane, which is normally constituted from the basement membranes of the RPE and choroidal cells. This led us to wonder whether or not the local coverage of RPE patches influences the polarity of retinal epithelial cell in their vicinity at early developmental stages in $n o k^{m 520}$. We thus examined the spatial relationship between the patches of the RPE and M-phase nuclei 
and apical marker ZO- 1 in $n o k^{m 520}$ retinas at $36 \mathrm{hpf}$. Interestingly, we found that the coverage of retina by RPE patches did not relieve the retinal polarity defects locally (Fig. 9D), suggesting RPE patches in $n o k^{m 520}$ are not equivalent to an intact RPE in pt106 in mediating retinal epithelial polarity.

The above results prompted us to ask whether the RPE integrity or the expression of wild-type Nok in the RPE cells is important to maintain retinal epithelial polarity. Previously, it was described that sporadic wild-type donor RPE cells might be able to restore the apical localization of moe mutant M-phase retinal nuclei by presumably secreting some signaling factors, as suggested by the over fourfold reduction of ectopic localization of moe $\mathrm{M}$-phase nuclei in the presence of sporadic wild-type RPE donor cells (Jensen et al., 2001). To investigate whether sporadic wild-type RPE cells can restore retinal epithelial polarity defects in $n o k^{m 520} \mathrm{mu}-$ tants, we transplanted wild-type pt104 blastomeres into nok ${ }^{m 520}$ mutants and examined the retinal regions that were adjacent to wild-type RPE donor cells. Surprisingly, in contrast to the results of Jensen et al. (2001), we found that the percentages of interiorly localized M-phase nuclei showed no apparent difference, whether or not there were wild-type RPE donor cells in the vicinity (Fig. 10) [when adjacent to wild-type RPE donor cells, $\sim 87.8 \%$ of the M-phase nuclei localized interiorly ( $n=41$ ); when distant from wild-type RPE donor cells, $\sim 89.3 \%$ of the M-phase nuclei localized interiorly $(n=47)]$. These percentages of ectopic nuclei were similar to that in the regular nok ${ }^{m 520}$ mutants (Fig. $5 D$ ). In addition, apical marker ZO-1 also localized interiorly in the host retina (Fig. 10, right panel, red). The inability of sporadic wild-type RPE cells to rescue retinal polarity defects is not likely attributable to the $n o k^{m 520}$ mutation, because the wild-type donor retinal cells also displayed the polarity defects whether or not they were adjacent to wild-type RPE donor cells (Figs. $8 B ; 10$, red arrow). Considering the restoration of retinal epithelial polarity in pt106 (Figs. 7, 8A), these results indicate that an intact wild-type RPE is required to maintain the proper retinal epithelial polarity, but sporadic wild-type RPE cells are not sufficient to do so.

\section{Discussion}

In summary, our study revealed a series of novel developmental steps by which the nok gene regulates retinal development through RPE-retina interactions (Fig. 11): The expression of Nok in the RPE is required and sufficient to maintain RPE integrity. An intact RPE ensures proper polarity of retinal neuroepithelium before photoreceptor genesis, and a properly polarized retinal epithelium ensures apical localization of cell divisions and provides a proper environment for postmitotic cell migration and patterning. Finally, although retinal expression of Nok is not re- quired for retinal cell polarity and patterning during the genesis of retinal ganglion cells and inner nuclear cells, it is required during embryonic photoreceptor genesis.

\section{The autonomy and nonautonomy of Nok function in the RPE} and the retina

In the RPE, Nok maintains its integrity in a tissue-autonomous manner. This autonomy resembles the way in which Nok homologs play a role in maintaining the polarity and integrity of the fly embryonic epithelium and MDCK cell monolayer (Tepass and Knust, 1993; Kamberov et al., 2000; Bachmann et al., 2001; Hong et al., 2001; Straight et al., 2004). In contrast, Nok cannot maintain proper retinal epithelial polarity in an autonomous manner (Fig. $8 A, B$ ). Such differential requirements of Nok suggest a mechanical variation in maintaining epithelial polarity and integrity of the RPE and the retina.

\section{Role of RPE in maintaining retinal epithelial polarity}

Sporadic wild-type donor RPE cells were suggested to be able to rescue the polarity defect of retinal cells in the moe mutant hosts (Jensen et al., 2001). However, our similar blastomere transplan- 

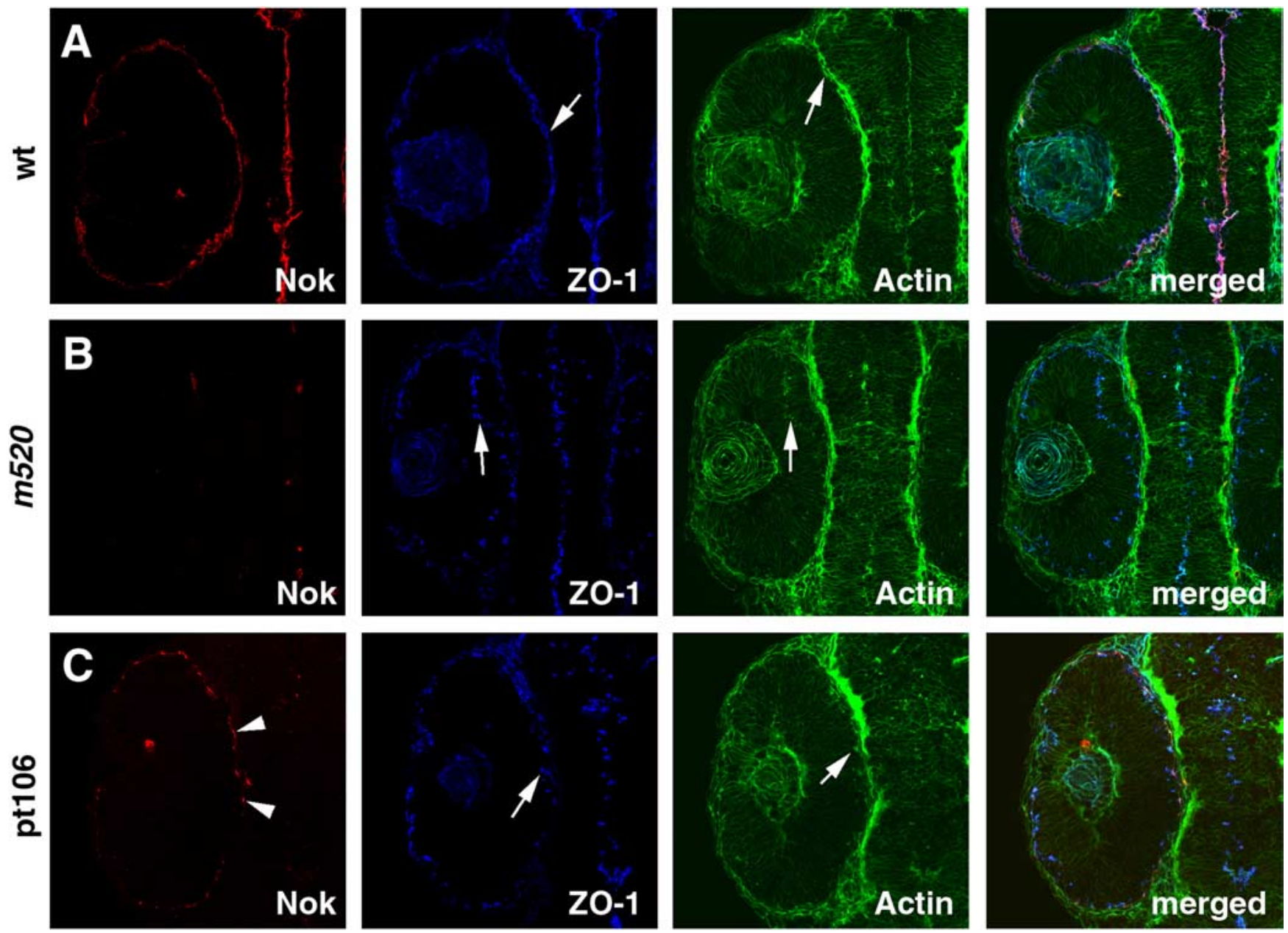

Figure 7. The retinal epithelial polarity is recovered in pt106 at early stages of retinal development. $\boldsymbol{A}-\boldsymbol{C}$, As in wild type $(\boldsymbol{A})$, transgenic expression of Nok (red; arrowheads) in the RPE restores the apical localization of apical markers Z0-1 (blue; arrows) and adherens junction-associated actin bundles (green; arrows) in pt106 retina at $36 \mathrm{hpf}$ ( $\boldsymbol{C}$. These apical markers localize ectopically to the interior of the retina in $n o k^{m 520}(\boldsymbol{B}$, arrows). Embryos were treated with PTU so that transgenic Nok expressed in the RPE would not be obscured by melanin pigmentation.
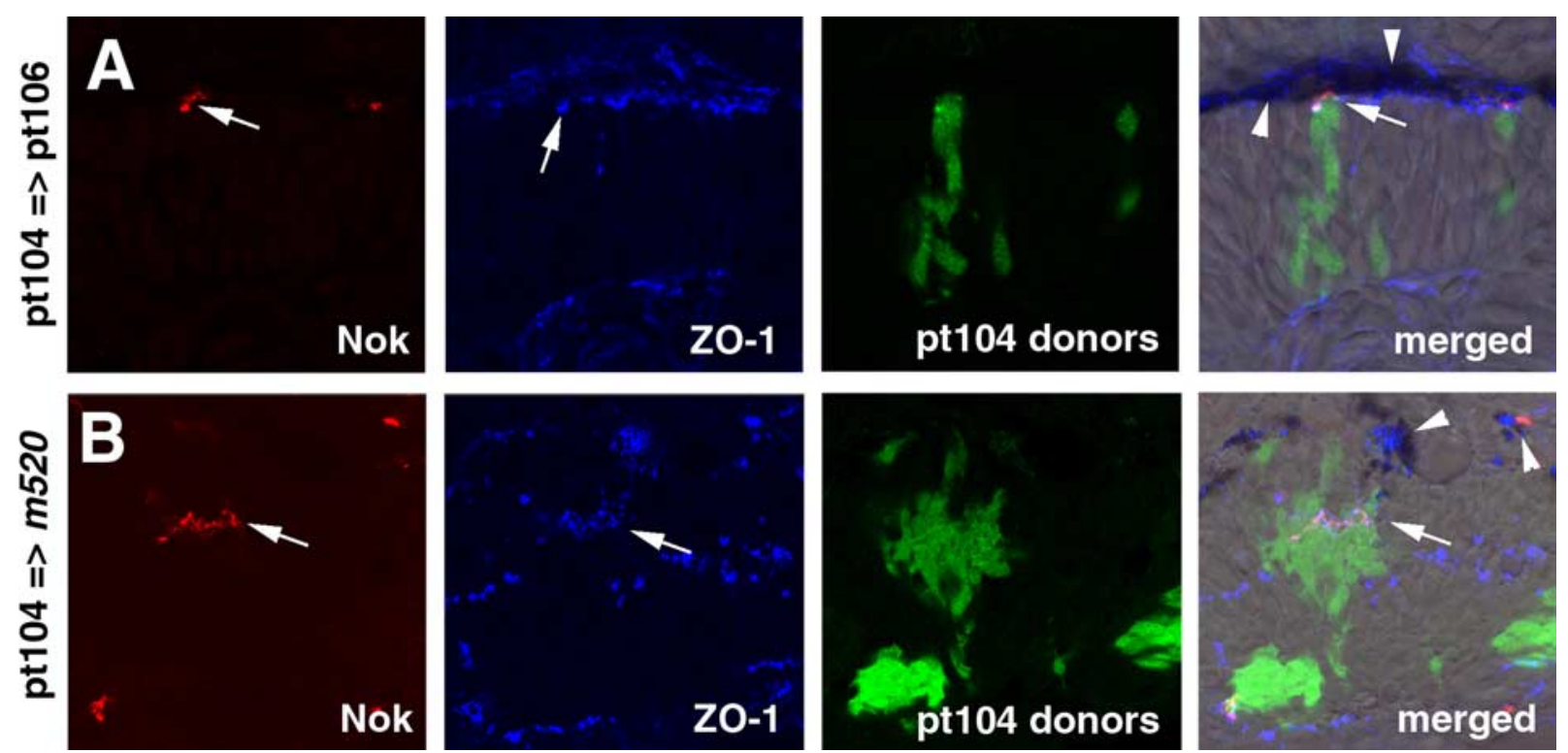

Figure 8. Retinal expression of wild-type Nok is not sufficient to rescue the retinal neuroepithelial polarity defect in $n o k^{m 520}$. $A$, Nok and Z0-1 expressed in the wild-type pt 104 donor cells localize apically in the pt 106 host retinas at $36 \mathrm{hpf}$ (arrows). The arrowheads indicate the intact RPE as revealed by differential interference contrast (DIC) imaging. B, Nok and Z0-1 expressed in the wild-type pt104 donor cells localize ectopically to the interior of the $n o k^{m 520}$ host retinas at $36 \mathrm{hpf}$ (arrows). The arrowheads indicate the fragmented patchy RPE as revealed by DIC imaging. The tissue sections were oriented with the RPE on top and the lens at bottom. 

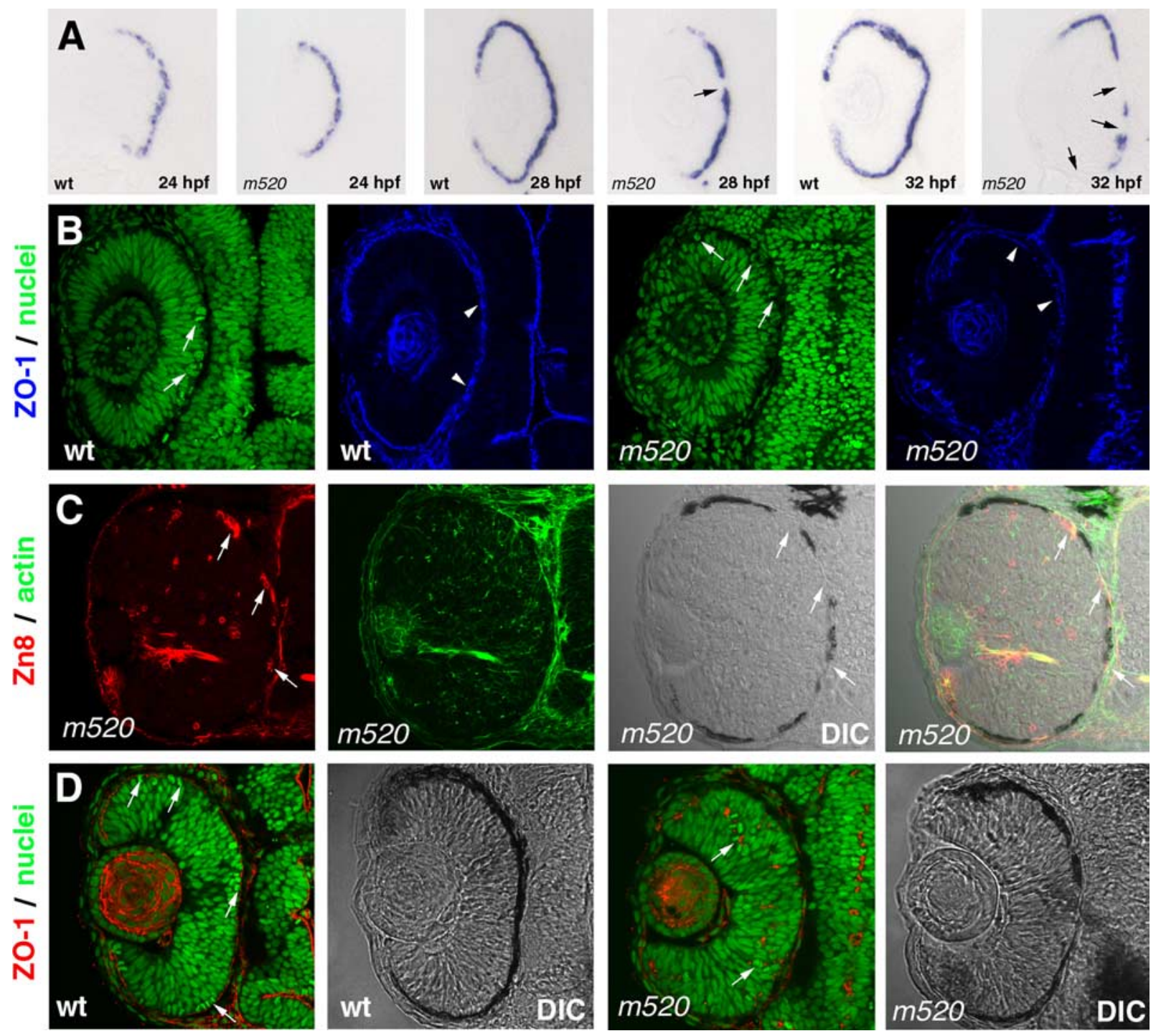

Figure 9. The disruption of retinal epithelial polarity in $n o k^{m 520}$ is correlated with the loss of RPE integrity in a tight temporal manner. $A$, An in situ hybridization analysis of wild-type and nok ${ }^{m 520}$ embryos with an antisense RNA probe against the zebrafish TYRP1 revealed the presence of an intact RPE structure in $n o k^{m 520}$ embryos at $24 \mathrm{hpf}$. The disruption of RPE integrity progressively worsens as retinal neurogenesis unfolds; the arrows indicate the disrupted RPE regions in $n o k^{m 520}$ at 28 and $32 \mathrm{hpf}$. B, At 26 hpf, the polarity of the retinal epithelium is still mostly unaffected by the nok ${ }^{m 520}$ mutation. Apical markers Z0-1 (blue; arrowheads) and M-phase nuclei (green; arrows) localize to the apical surface in both wild-type and nok ${ }^{\mathrm{m} 520}$ mutant retinas. C, At 48 hpf in nok ${ }^{\mathrm{m} 520}$, ectopic retinal ganglion cells (red; Zn8 staining; arrows) prefer to accumulate at the apical retinal regions adjacent to disrupted RPE areas [differential interference contrast (DIC) imaging]. Actin staining (green) is for the background morphology. D, At 36 hpf, the ectopic localization of M-phase nuclei (green; Y0-PRO; arrows) and Z0-1 maker (red; arrows) in nok ${ }^{\mathrm{m} 520}$ is not influenced by local presence or absence of the RPE patches (right two panels). The left two panels are controls for wild type.

tation analysis in the nok background did not support such a capability (Fig. 10). The reasons for this discrepancy are unclear. We cannot rule out the possibility that different mutation background made the difference. In fact, unlike the apical localization of Nok, the distribution of Moe to the entire cell membrane suggests that the molecular mechanisms by which Moe regulates retinal epithelial polarity are different from that for Nok (Hsu et al., 2006). Alternatively, because moe mutation does not cause a severe retinal polarity defect at early developmental stages (Jensen et al., 2001, their Fig. 3F) (data not shown), it might be challenging to assess to what extent sporadic wild-type RPE cells really restored the apical localization of $\mathrm{M}$-phase nuclei in moe. Thus, to our knowledge, our study is the first to demonstrate with a stable genetic system that retinal epithelial polarity is dependent on an intact RPE, and sporadic wild-type RPE cells are not sufficient to regulate retinal epithelial polarity.

Although the RPE can rescue the retinal epithelial polarity defect in pt106 before embryonic photoreceptor genesis, the later loss of retinal epithelial polarity indicates that retinal expression of Nok is still required to sustain the polarity during the period that embryonic photoreceptors are generated (Fig. 5). Thus, the requirement of the RPE for retinal epithelial polarity and retinal cellular pattern formation gradually decreases during development.

\section{Mechanisms of the maintenance of retinal epithelial polarity by RPE}

There are several possible mechanisms by which the RPE maintains retinal epithelial polarity before embryonic photoreceptor genesis. During the formation of the optic cup in zebrafish, the apical surfaces faces of the RPE and the retina juxtapose tightly with each other (Schmitt and Dowling, 1994). It is likely that there is a physical adhesion between the apical surfaces of the two epithelia. This potential adhesion may serve as a pulling force to maintain the polarity of the retinal epithelium at early stages of 
donor cells

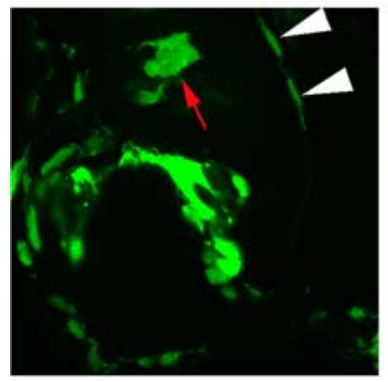

nuclei

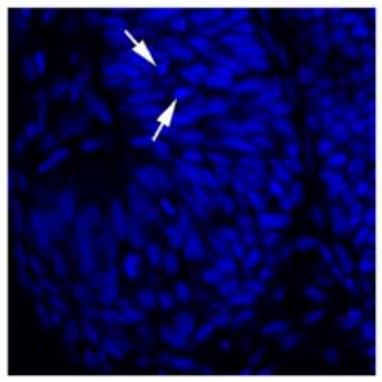

merged / ZO-1

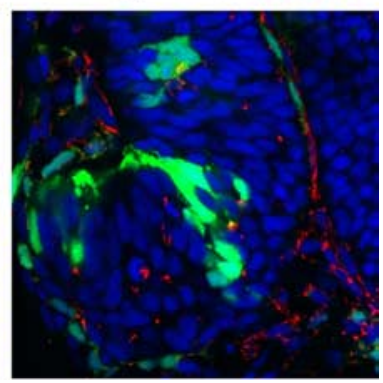

Figure 10. Sporadic wild-type RPE cells were not sufficient to rescue the retinal epithelial polarity. Wild-type pt104 donor cells (visualized by GFP; green) were transplanted into $n o k^{m 520}$ mutants. The localization of retinal M-phase nuclei (white arrows; blue TO-PRO staining for the nuclei) and the apical marker Z0-1 (red) were analyzed at $36 \mathrm{hpf}$. Near the regions that contained wild-type RPE cells (arrowheads), both wild-type retinal donor cells (red arrows) and host retinal cells displayed an identical polarity defect of interior localized M-phase nuclei and apical marker Z0-1 (red), as did the retinal cells that were distant from the wild-type RPE donor cells or in pure nok ${ }^{m 520}$ mutants (for statistical analysis, see Results).

development. The loss of RPE integrity, as caused in $n o k^{m 520}$, may reduce the adhesion between the two tissues, leading to the loss of retinal epithelial polarity. This hypothesis is consistent with the tight temporal association between the patchy RPE defect and retinal polarity defect in $n o k^{m 520}$ (Fig. 9) (data not shown). However, the molecular nature of this proposed physical adhesion is not known. A previous EM observation suggested that desmosomes may play a role in the adhesion of the two tissues in human embryos (Hollenberg and Spira, 1973). Our EM and immunohistochemical analyses, however, revealed no apparent subcellular structures that resemble conventional cell-cell junctional complexes, such as desmosomes, between the two tissues in zebrafish embryos (data not shown).

The close contact between the RPE and the retina is essential but not sufficient to prove that the RPE regulates retinal epithelial polarity through physical adhesion. Other alternative possibilities warrant additional discussion here. First, the RPE may secrete diffusible factors (Vollmer et al., 1984; Rothermel et al., 1997; Layer et al., 1998; Nakagawa et al., 2003) to mediate retinal epithelial polarity. Although the inability of the sporadic wild-type RPE cells to rescue the polarity defect of their neighbor $n o k^{m 520}$ retinal cells (Fig. 10) does not favor this secreted-factor theory, it cannot rule it out either. This is because the sufficient secretion of any RPE factors may require a properly polarized intact RPE tissue. Sporadic wild-type RPE donor cells may not be able to make proper connections with neighboring host mutant RPE cells, leading to the loss of their secretion function. Or it could be that a few sporadic wild-type RPE donor cells cannot constitute the critical mass needed to secrete enough factors for proper functions. Second, the RPE may block some polarity-disrupting signals from the Bruch's membrane or the choroid, just as the RPE prevents retinal ganglion cells from migrating incorrectly to the apical retinal regions (Zolessi et al., 2006) (Fig. 9C). However, this possibility is not supported by the fact that the retinal polarity defect exists in all retinal regions in $n o k^{m 520}$ mutants regardless of whether or not there are RPE cells nearby (Fig. 9D).

\section{The functions of retinal Nok}

Previously, we showed that the adherens junctions in the retina are likely the precursor of the OLM, and Nok plays a role in the development and maintenance of the OLM (Wei et al., 2006b). These findings are further supported by the current observation that the OLM does not develop in pt106, which lacks retinal Nok function (Fig. 4). It is likely that the disruption of Nok-mediated photoreceptor-photoreceptor adhesion/ stabilization is one of the underlying causes of the improper positioning of photoreceptors in pt106. The Nok is required to target Crumbs to the inner segment region to potentially mediate physical adhesion via the extracellular domain of the Crumbs proteins (Wei et al., 2006b). Consistent with this notion, we found that Crumbs is internalized in photoreceptors in pt106 as in regular nok $k^{m 520}$ mutants (supplemental Fig. 1, available at www. jneurosci.org as supplemental material) (Wei et al., 2006b). Furthermore, the decline of apical cell division and the worsening of retinal polarity in pt 106 retina occur immediately before embryonic photoreceptor genesis (Fig. 5D) (Hu and Easter, 1999). This suggests that Nok also plays an important role in the transition of the adherens junctions between undifferentiated retinal epithelial cells to the OLM in the outer nuclear layer. Nevertheless, during the genesis of ganglion cells and inner nuclear cells, the maintenance of retinal epithelial polarity does not require retinal Nok (Fig. 5).

\section{Regulations of cell migration for retinal pattern formation}

As for how cell migration underlies cellular patterning process of the retina, our study suggests several aspects, which might be mutually inclusive. First, when M-phase cells are ectopically positioned in the interior of the retina, as in $n o k^{m 520}$, postmitotic cells are liable to migrate in abnormal directions, rather than the basal direction in wild type (supplemental movie 2, available at www.jneurosci.org as supplemental material; Fig. 6). Second, the undifferentiated retinal neuroepithelial cells that span the entire thickness of the retina may serve as a guiding mechanism for differentiating retinal cells by providing a continuous surface for postmitotic cells to adhere to during migration, or by providing proper migration cues. In $n o k^{m 520}$, the splitting of the mutant retinal epithelium into two halves (Figs. 5, 7, 8), with the outer one-half displaying a reversed apical-basal polarity compared with the adjacent inner one-half, will likely present confusing migration guidance cues to postmitotic cells, leading to irregular cellular movements (for comparison, see supplemental movies 1 and 2, available at www.jneurosci.org as supplemental material). Consistent with this hypothesis, in pt106, the degree to which a given type of retinal cell is properly positioned is positively correlated with the extent of the restoration of retinal epithelial polarity during the developmental stage when those cells are predominantly being generated (Figs. 4,5 ). In addition, as shown by Zolessi et al. (2006), the RPE might block the Bruch's membrane from attracting retinal ganglion cells to migrate to the apical end (Fig. 9C). Thus, the disruption of RPE integrity will further worsen the patterning process in $n o k^{m 520}$. However, the blockage function of RPE may not be sufficient/applicable for photoreceptors, because they localize improperly in pt106, even with an intact RPE during embryonic photoreceptor genesis (Fig. 4).

\section{Mosaic analyses by blastomere transplantation versus transgenesis}

Blastomere transplantation technology has been used by many researchers to study the autonomy of gene function. Although this technology is powerful and convenient, its strength can be limited if multiple types of cell-cell interactions exist simulta- 

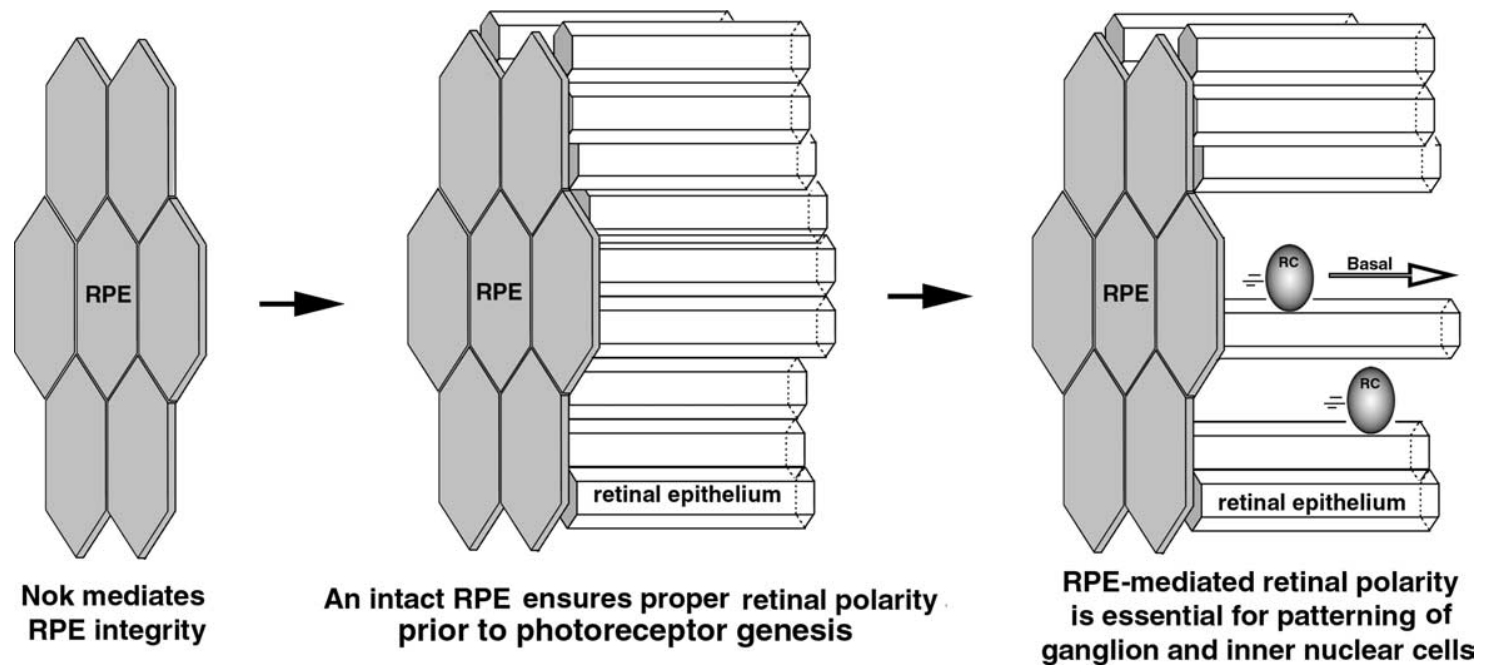

Figure 11. A model illustrates the steps by which the Nok-mediated intact RPE plays its essential role in retinal development (for a detailed explanation, see first paragraph of Discussion). RC stands for postmitotic migrating retinal cells.

neously in the mosaic animals. Thus, uncontrollable distribution of donor cells can cause ambiguity when one tries to determine whether a particular cell-cell interaction causes certain effects. This limitation manifests itself when determining whether the extrinsic regulation of the patterning of individual retinal cells is directed by the RPE or by the neighboring retinal cells (Jensen et al., 2001; Wei and Malicki, 2002; Zolessi et al., 2006). This limitation can be dramatically overcome by using transgenic models, in which gene expression/function in desired cells or tissues can be manipulated in a stable and reproducible manner.

The transgenic approach has its own limitations as well: Proper promoters for cell- or tissue-specific expression of the transgenes might not be available. In addition, the position effects of transgenesis may alter the desired expression patterns in certain transgenic lines, so it requires vigorous selection and verification to obtain desired transgenic lines. To study the requirement of the RPE for retinal development, Raymond and Jackson (1995) ablated the RPE via transgenic expression of the toxic protein diphtheria toxin A in two transgenic lines: line A (anophthalmic) and line $\mathrm{M}$ (microphthalmic). The brain abnormality and the earlier-than-expected onset of retinal defect in line A at embryonic day 11 (E11) raised concern of ectopic retinal expression of the toxic transgene (the study unfortunately did not verify the tissue specificity of the transgenic expression). The late onset of RPE ablation in line M, at E14.5, when the retina had already developed nearly perfect lamination of inner and outer neuroblastic layers, made line M unsuitable for revealing RPE functions during early retinal development. In contrast, the restoration of the RPE in pt106 preserves the neurotrophic function of the RPE for retinal cell survival (Ishida et al., 1997) and establishes pt106 as a unique system for characterizing the roles of RPE in retinal epithelial polarity at early developmental stages and in cellular pattern formation during retinal neurogenesis.

\section{References}

Bachmann A, Schneider M, Theilenberg E, Grawe F, Knust E (2001) Drosophila Stardust is a partner of Crumbs in the control of epithelial cell polarity. Nature 414:638-643.

Baye LM, Link BA (2007) Interkinetic nuclear migration and the selection of neurogenic cell divisions during vertebrate retinogenesis. J Neurosci 27:10143-10152.

Camacho-Hübner A, Rossier A, Beermann F (2000) The Fugu rubripes ty- rosinase gene promoter targets transgene expression to pigment cells in the mouse. Genesis 28:99-105.

Catalano AE, Raymond PA, Goldman D, Wei X (2007) Zebrafish dou yan mutation causes patterning defects and extensive cell death in the retina. Dev Dyn 236:1295-1306.

Das T, Payer B, Cayouette M, Harris WA (2003) In vivo time-lapse imaging of cell divisions during neurogenesis in the developing zebrafish retina. Neuron 37:597-609.

Dowling JE (1970) Organization of vertebrate retinas. Invest Ophthalmol 9:655-680.

Erdmann B, Kirsch FP, Rathjen FG, Moré MI (2003) N-cadherin is essential for retinal lamination in the zebrafish. Dev Dyn 226:570-577.

Funke L, Dakoji S, Bredt DS (2005) Membrane-associated guanylate kinases regulate adhesion and plasticity at cell junctions. Annu Rev Biochem 74:219-245.

Hinds J, Hinds P (1974) Early ganglion cell differentiation in the mouse retina: an electron microscopic analysis utilizing serial sections. Dev Biol 37:381-416.

Ho RK, Kane DA (1990) Cell-autonomous action of zebrafish spt-1 mutation in specific mesodermal precursors. Nature 348:728-730.

Hollenberg MJ, Spira AW (1973) Human retinal development: ultrastructure of the outer retina. Am J Anat 137:357-385.

Hong Y, Stronach B, Perrimon N, Jan LY, Jan YN (2001) Drosophila Stardust interacts with Crumbs to control polarity of epithelia but not neuroblasts. Nature 414:634-638.

Hsu YC, Willoughby JJ, Christensen AK, Jensen AM (2006) Mosaic Eyes is a novel component of the Crumbs complex and negatively regulates photoreceptor apical size. Development 133:4849-4859.

Hu M, Easter SS (1999) Retinal neurogenesis: the formation of the initial central patch of postmitotic cells. Dev Biol 207:309-321.

Ishida K, Yoshimura N, Yoshida M, Honda Y, Murase K, Hayashi K (1997) Expression of neurotrophic factors in cultured human retinal pigment epithelial cells. Curr Eye Res 16:96-101.

Jensen AM, Westerfield M (2004) Zebrafish mosaic eyes is a novel FERM protein required for retinal lamination and retinal pigmented epithelial tight junction formation. Curr Biol 14:711-717.

Jensen AM, Walker C, Westerfield M (2001) mosaic eyes: a zebrafish gene required in pigmented epithelium for apical localization of retinal cell division and lamination. Development 128:95-105.

Kamberov E, Makarova O, Roh M, Liu A, Karnak D, Straight S, Margolis B (2000) Molecular cloning and characterization of Pals, proteins associated with mLin-7. J Biol Chem 275:11425-11431.

Layer PG, Rothermel A, Willbold E (1998) Inductive effects of the retinal pigmented epithelium (RPE) on histogenesis of the avian retina as revealed by retinospheroid technology. Semin Cell Dev Biol 9:257-262.

Malicki J, Jo H, Pujic Z (2003) Zebrafish N-cadherin, encoded by the glass 
onion locus, plays an essential role in retinal patterning. Dev Biol 259:95-108.

Nakagawa S, Takada S, Takada R, Takeichi M (2003) Identification of the laminar-inducing factor: Wnt-signal from the anterior rim induces correct laminar formation of the neural retina in vitro. Dev Biol 260:414-425.

Pauls S, Geldmacher-Voss B, Campos-Ortega JA (2001) A zebrafish histone variant H2A.F/Z and a transgenic H2A.F/Z:GFP fusion protein for in vivo studies of embryonic development. Dev Genes Evol 211:603-610.

Raymond SM, Jackson IJ (1995) The retinal pigmented epithelium is required for development and maintenance of the mouse neural retina. Curr Biol 5:1286-1295.

Rothermel A, Willbold E, Degrip WJ, Layer PG (1997) Pigmented epithelium induces complete retinal reconstitution from dispersed embryonic chick retinae in reaggregation culture. Proc R Soc Lond B Biol Sci 264:1293-1302.

Schmitt EA, Dowling JE (1994) Early eye morphogenesis in the zebrafish, Brachydanio rerio. J Comp Neurol 344:532-542.

Straight SW, Shin K, Fogg VC, Fan S, Liu CJ, Roh M, Margolis B (2004) Loss of PALS1 expression leads to tight junction and polarity defects. Mol Biol Cell 15:1981-1990.
Tepass U, Knust E (1993) Crumbs and stardust act in a genetic pathway that controls the organization of epithelia in Drosophila melanogaster. Dev Biol 159:311-326.

Vollmer G, Layer PG, Gierer A (1984) Reaggregation of embryonic chick retina cells: pigment epithelial cells induce a high order of stratification. Neurosci Lett 48:191-196.

Wei X, Malicki J (2002) nagie oko, encoding a MAGUK-family protein, is essential for cellular patterning of the retina. Nat Genet 31:150-157.

Wei X, Luo Y, Hyde DR (2006a) Molecular cloning of three zebrafish lin7 genes and their expression patterns in the retina. Exp Eye Res 82:122-131.

Wei X, Zou J, Takechi M, Kawamura S, Li L (2006b) Nok plays an essential role in maintaining the integrity of the outer nuclear layer in the zebrafish retina. Exp Eye Res 83:31-44.

Zolessi FR, Poggi L, Wilkinson CJ, Chien CB, Harris WA (2006) Polarization and orientation of retinal ganglion cells in vivo. Neural Develop 1:2

Zou J, Beermann F, Wang J, Kawakami K, Wei X (2006) The Fugu tyrp1 promoter directs specific GFP expression in zebrafish: tools to study the RPE and the neural crest-derived melanophores. Pigment Cell Res 19: $615-627$. 